\title{
Le tramway a-t-il guidé la croissance urbaine au XIXème s. ? L'exemple de Nancy
}

Did the tramway network guide urban growth in the 19th century? Case study of Nancy

Hat die Straßenbahn das städtische Wachstum im 19. Jahrhundert geleitet? Das Beispiel von Nancy

\section{Anne Hecker}

\section{OpenEdition}

\section{Journals}

Édition électronique

URL : http://journals.openedition.org/rge/7243

ISSN : 2108-6478

Éditeur

Association des géographes de l'Est

Référence électronique

Anne Hecker, «Le tramway a-t-il guidé la croissance urbaine au XIXème s. ? L'exemple de Nancy », Revue Géographique de l'Est [En ligne], vol. 57 / 3-4 | 2017, mis en ligne le 28 septembre 2018, consulté le 06 octobre 2018. URL : http://journals.openedition.org/rge/7243

Ce document a été généré automatiquement le 6 octobre 2018.

Tous droits réservés 


\section{Le tramway a-t-il guidé la croissance urbaine au XIXème s. ? L'exemple de Nancy}

Did the tramway network guide urban growth in the 19th century? Case study of Nancy

Hat die Straßenbahn das städtische Wachstum im 19. Jahrhundert geleitet? Das Beispiel von Nancy

\section{Anne Hecker}

1 La loi Solidarité et Renouvellement Urbains (SRU, 2000) a mis au cœur de l'aménagement le principe d'une croissance de l'espace urbain, dont les extensions nouvelles seraient prioritairement adhérentes aux axes de transport collectif. La limitation du phénomène d'étalement, souvent peu contrôlé depuis l'ouverture de la ville facilitée par les modes motorisés, est ainsi mise entre les mains des documents d'urbanisme, à commencer par les SCoT, chargés de favoriser cette orientation des constructions vers des corridors bien desservis. La signature de contrats d'axe entre les autorités organisatrices des transports urbains, les collectivités locales et les entreprises qui le mettent en œuvre, à l'image de Grenoble ou de Toulouse, représente un des aboutissements possibles de cette démarche (Bentayou et al. 2015, Maulat 2015), pourtant souvent

2 Dès lors, un principe implicite émerge en creux de cette orientation de l'urbanisme en fonction des transports collectifs : celui de la nécessaire simultanéité de l'axe avec la constitution de l'espace urbain, voire de son antériorité. Cette co-construction implique un important changement de procédures au regard du fonctionnement actuel en matière de création d'axes lourds - ou pourrait même évoquer un changement de paradigme en matière de transports structurants (Delpirou 2009, Gallez et Chardonnel 2018). Depuis le retour du tramway dans les années 1980 , le coût de ces réalisations, et la recherche d'une hypothétique rentabilité économique ont davantage guidé ces réalisations vers les espaces de fortes densités et concentrant les pôles générateurs et attracteurs de trafic 
(Zembri 2012), que vers les espaces présentant de réelles opportunités foncières, mais devant encore être développés.

3 La possibilité d'une telle adhérence de l'urbanisation aux infrastructures de transport (Vigarié 1983, Desjardins et al. 2011) invoque alors une potentielle capacité structurante de ces dernières. Les études américaines s'intéressent ainsi aux formes urbaines nées du train et du tramway, mais aussi aux différentiels de densité encore sensibles actuellement autour de leurs arrêts, et aux comportements de mobilité spécifiques hérités des Tracked Cities et Streetcar Suburbs (Burchfield et al. 2006; Block-Schachter 2012; Brooks et Lutz 2016 ; Warner 1978). Elles seraient donc à même, sinon de faire naître la ville, du moins de la guider - ainsi, le tramway précéderait « les espaces urbains futurs en acquérant le statut de pivot organisateur de la trame urbaine " (Redondo 2012, IPRAUS-ENSAPB, 2005). Cette idée est évoquée pour la croissance urbaine qui, au XIXème et début XXème siècle, aurait prioritairement adhéré aux infrastructures de transport lourdes, façonnant une forme urbaine en grains de chapelet et en doigts de gant (Merlin 1982, Schaeffer et Sclar 1980).

Une question pourtant souvent débattue, puisque le principe d'effets structurants des infrastructures de transport a été remis en cause pour les axes modernes (Offner 1993), celui de l'impact des voies de chemins de fer du XIXème sur la hiérarchie urbaine a été bousculé (Pumain 1982) et la réalité de l'adhérence ferroviaire actuelle n'est pas encore totalement acquise (Desjardins 2011, Maulat 2015, Meunier-Chabert et Trêve-Thomas 2018). On peut donc s'interroger sur la capacité structurante des voies de tramways qualifiées d'historiques, sur l'intensité du lien qui se serait tissé entre elles et l'avancée du front urbain. En effet, ces longs réseaux de tramway créés fin XIXème et ayant parcouru de nombreuses villes et banlieues jusque dans les années 1960, se sont fortement développés au moment où exode rural et révolution industrielle imposaient aux villes d'accueillir une population en forte croissance, et où la densification de l'espace déjà construit laissait place à l'évolution de la forme urbaine, sous l'impact d'une mobilité facilitée par les transports en commun.

deja au début du XXème siècles, des chercheurs s'interrogent sur le rôle que ces infrastructures jouent sur la ville, sur leur capacité à en accroître la population, à modifier la superficie de l'agglomération ou la répartition des habitants et des activités à l'intérieur de celle-ci (Calvayrac 1913). Avec le recul que le temps permet, on peut reprendre ces interrogations au regard de l'évolution de la ville. Le tramway a-t-il eu un rôle "préventif ", en précédant la construction des nouveaux secteurs de la ville, ou endosse-t-il un rôle "curatif » en desservant des secteurs déjà densifiés et recadrant a posteriori l'urbanisme (Woessner et Lanneaux, 2012)? On s'interroge alors sur un éventuel rôle que le réseau aurait pu avoir sur la constitution de la forme urbaine au XIXème et au début du XXème siècle, sur sa capacité à jouer un rôle attractif, à même de favoriser la localisation des habitations nouvelles le long d'axes privilégiés, au détriment d'espaces en creux, plus éloignés des voies desservies. On peut également questionner son influence sur la différenciation des quartiers. Bien avant les politiques de zonage du second XXème siècle, a-t-il pu contribuer à spécialiser socialement et fonctionnellement certaines zones de la ville?

6 Aujourd'hui, l'épaisseur du temps rend possible la réalisation d'un bilan de l'impact de ces lignes historiques, tant à l'époque de leur mise en œuvre et dans les années qui ont suivi, que sur le long terme. Il rend toutefois plus complexe la lecture de la ville, dont les strates se sont accumulées et peuvent brouiller la lecture des lieux. Afin d'apporter des éléments de réflexion sur l'influence du tramway historique sur la ville de l'époque mais aussi 
actuelle, une vaste étude est en cours, portant sur le cas de la ville de Nancy, et mettant en œuvre un outil d'aide à la réflexion, un SIG historique. Mobilisant plans anciens et documents d'archives, leur intégration dans cet outil permet de reconstituer l'avancée pas à pas de la ville, et d'y mesurer l'impact du tramway. Nous en évoquerons les apports, avant de nous intéresser à l'intégration du tramway dans la ville et à son impact sur la forme de celle-ci.

\section{Terrain d'étude et méthodologie -un SIG historique au service de la compréhension du lien urbanisation tramway à Nancy}

7 De nombreux documents, cartographiques et/ ou historiques, nous renseignent sur l'évolution des villes et sur les réseaux de transport, notamment collectifs. Néanmoins, à l'état brut, les informations collectées dressent un tableau général, mais où manque la finesse de l'information et de la spatialisation, à même de répondre à nos interrogations sur le rôle des infrastructures dans la ville. Dans l'objectif d'apporter des éléments tangibles et précis sur la réalité du caractère structurant de ce réseau, un SIG historique est en cours de constitution sur le cas de l'agglomération de Nancy. L'évolution entre les années 1870 à 1895 , période de très forte croissance urbaine, a été particulièrement investie, et sera ici exploitée pour mesurer le rôle joué par les lignes de tramway dans l'avancée de la tache urbaine.

\section{A- Nancy, un vaste réseau au service d'une forte croissance urbaine}

8 Le choix de l'agglomération de Nancy pour l'étude du lien entre les anciens réseaux de tramway et la croissance de la ville s'avère doublement judicieux. Elle appartient en effet aux villes moyennes ayant implanté précocement un réseau historique, dès 1874 pour sa première ligne, alors hippomobile. En France, seules quatorze villes créent leurs premières lignes avant 1880 (Robert 1974), Nancy relève donc des villes pionnières pour ce nouveau mode de transport collectif. Un premier réseau (4 lignes, 13,6 km) voit le jour avant la fin du XIXème siècle. Un second réseau naît avec l'électrification début XXème, qui insuffle un nouveau souffle et voit naître de multiples projets (plus de quinze nouveaux kilomètres envisagés dès 1902 !). Modernisé, électrifié, il s'accroît régulièrement, et atteint déjà $27 \mathrm{~km}$ en 1911. Il prend alors son essor, les lignes urbaines se multiplient, puis s'étendent vers les communes de banlieue. Un réseau périurbain de près de trente kilomètres à même de structurer ces espaces périphériques complète les lignes urbaines, circulant à travers des espaces parfois encore peu habités. A son apogée, le réseau a compté jusqu'à près de $70 \mathrm{~km}$ en service pour 120000 habitants. Quelques compléments seront encore construits dans les années 1930, mais parallèlement, les premières liaisons déficitaires disparaissent, remplacées par des autobus. À l'instar de l'essentiel des agglomérations, le réseau sera démantelé à l'aube des années 1960.

9 Fin XIXème s., le réseau de tramway grandissant offre un service nouveau à une population elle-même en forte croissance. Au regard des autres villes qui enregistrent alors une poussée urbaine bien connue, particulièrement due à la révolution industrielle combinée à l'exode rural, Nancy doit absorber rapidement un second apport de population spécifique, lié à la fin du conflit de 1870. L'annexion de la Moselle et de 
l'Alsace à l'Empire allemand provoque à Nancy un afflux de population en provenance de ces départements. On doit à ces "optants » une transformation de l'espace urbain, liée tant à l'accroissement manifeste du nombre d'habitants qu'à la création de fonctions urbaines inédites. Ainsi, un secteur d'activité industrielle se développe, au sein d'une cité jusqu'à présent davantage "ville d'études en même temps que de luxe et de mœurs élégantes " ${ }^{1}$ qu'ouvrière. Déplacement d'entreprises depuis les régions annexées, dédoublement de leurs activités ou création nouvelles, une activité manufacturière se développe, incitant une population ouvrière rurale à migrer vers Nancy (Sicard - Lenattier 2000), à l'origine de l'accroissement et de la transformation des quartiers à l'est de la ville. Certaines de ces entreprises ont acquis une forte notoriété telles que l'imprimerie Berger-Levrault ou les verreries Daum. Cette dernière est à l'origine du mouvement d'Art Nouveau dit « Ecole de Nancy ", dont l'épanouissement marque également la ville dans son architecture et ses décors.

Dès lors, l'accroissement de la population s'avère particulièrement rapide dans les années qui suivent le conflit, ainsi que l'illustrent les données issues des recensements de population.

Tableau 1 - Population et bâti à Nancy : 1869 - 1896

\begin{tabular}{|c|c|c|c|c|c|c|}
\hline & \multicolumn{2}{|c|}{ Habitants } & \multicolumn{2}{|l|}{ Maisons } & \multicolumn{2}{|c|}{ Logements occupés } \\
\hline & Nombre & progression & Nombre & progression & Nombre & progression \\
\hline 1869 & 46538 & & & & & \\
\hline 1872 & 50567 & $8,66 \%$ & 4196 & & 12812 & \multirow{3}{*}{$61,99 \%$} \\
\hline 1876 & 60755 & $20,15 \%$ & 4853 & $15,66 \%$ & & \\
\hline 1881 & 66056 & $8,73 \%$ & 4986 & $2,74 \%$ & 20754 & \\
\hline 1886 & 69481 & $5,18 \%$ & 5488 & $10,07 \%$ & 21795 & $5,02 \%$ \\
\hline 1891 & 75590 & $8,79 \%$ & 6232 & $13,56 \%$ & 24237 & $11,20 \%$ \\
\hline 1896 & 83634 & $10,64 \%$ & 7177 & $15,16 \%$ & 27455 & $13,28 \%$ \\
\hline
\end{tabular}

Sources : Recensements de population

Tableau 2 -Evolution du bâti et des habitants par section, entre 1872 et 1886 (en \%)

\begin{tabular}{|l|l|l|l|l|}
\hline & Sections & Immeubles & Population & Individus / immeuble \\
\hline \multirow{5}{*}{ Intra-muros } & 2 & $-11,6$ & $-5,3$ & $+7,2$ \\
\cline { 2 - 5 } & 4 & $-15,1$ & $+12,5$ & $+32,6$ \\
\cline { 2 - 6 } & 5 & $-4,2$ & $+3,9$ & $+8,5$ \\
\cline { 2 - 5 } & 7 & $-12,1$ & $+8,2$ & $+23,01$ \\
\hline
\end{tabular}




\begin{tabular}{|l|l|l|l|l|}
\hline \multirow{4}{*}{ Extérieures } & $\mathbf{1}$ (est) & $+98,3$ & $+127,3$ & $+14,6$ \\
\cline { 2 - 5 } & $\mathbf{3}$ (sud-ouest) & $+75,6$ & $+140,1$ & $+36,7$ \\
\cline { 2 - 5 } & $\mathbf{6}$ (ouest) & $+64,2$ & $+71,5$ & $+4,5$ \\
\cline { 2 - 5 } & $\mathbf{8}$ (nord-ouest) & $+33,6$ & $+52,5$ & $+14,1$ \\
\hline
\end{tabular}

Sources : recensements de population

Entre 1869 et 1886, la population de la ville de Nancy s'accroit ainsi de près de $50 \%$, gagnant notablement 10000 habitants en l'espace de 4 ans (1872 - 1876). Mais on le constate (tableaux 1), surtout au début de cette période de fort afflux de population, le nombre de logements s'accroît bien plus vite que celui des bâtiments. La densification et la division de l'espace au sein du bâti existant constituent une réponse d'urgence, qui décale la vague de croissance urbaine dans le temps. Mais finalement, le nombre d'immeubles construits gagne $71 \%$ entre 1872 et 1896, une croissance légèrement supérieure à celle du nombre d'habitants $(65 \%$ - cf tableau 1 , sources recensements de population).

12 Cet afflux de population se répartit de manière inégale dans la ville (Tableau 2). Les sections centrales, anciennement intra-muros, ne peuvent en accueillir qu'une part réduite en raison d'un bâti déjà dense, et de bâtiments anciens ne pouvant accroître leur population qu'à la marge (Collot 1980). Pour absorber le surplus de population, l'urbanisation nouvelle franchit alors le double carcan qui définissait jusqu'alors la ville : celui des fortifications des villes vieille (médiévale et Renaissance) et neuve (XVI XVIIème s.), déjà largement démantelées mais dont le tracé demeure prégnant dans le plan urbain; et au-delà, le carcan infrastructurel hérité du Second Empire, matérialisé par la voie ferrée à l'ouest et par le canal de la Marne-au-Rhin à l'est (Figure 1). Ce sont donc les sections extérieures, où l'espace est disponible, que population et bâtiments nouveaux investissent le plus fortement, particulièrement à l'est et au sud-ouest (sections 1 - partiellement intra-muros - 3 et 6 , cf figure 1 et Tableau 2). 


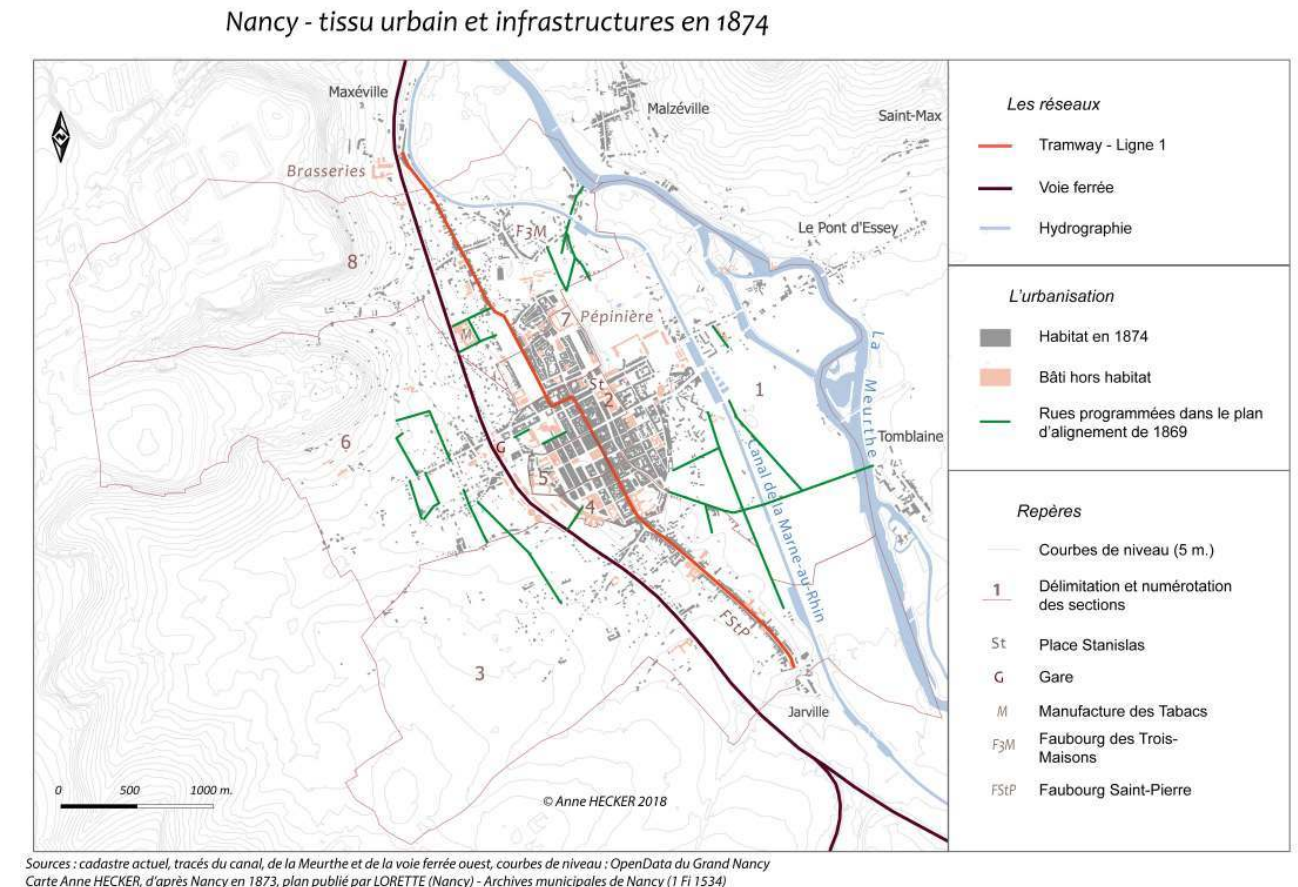

Les nouvelles extensions urbaines, qui absorbent l'essentiel de cet afflux de population, investissent alors des espaces encore largement ruraux, dénués de toute infrastructure, notamment de transport. Deux réalités contradictoires prévalent alors : d'une part celle des projets municipaux, qui définissent les tracés des nouvelles rues, que complète d'autre part une croissance plus spontanée, souvent hors de tout cadre légal. En effet, deux textes majeurs confient aux municipalités la maitrise de l'évolution de leur territoire. L'article 52 de la loi de 1807 impose ainsi les plans d'alignement, notamment « pour l'ouverture des nouvelles rues, pour l'élargissement des anciennes qui ne font pas partie d'une grande route». Le pouvoir d'alignement est donné aux maires, "conformément aux plans dont les projets auront été adressés au préfet, transmis avec leur avis au Ministère de l'Intérieur, et arrêtés au Conseil d'Etat». Il se complète en 1813 d'une circulaire interministérielle qui encadre le percement des rues nouvelles, "levier principal de la transformation des villes" (Laisney 2002), soumis au contrôle administratif central.

À Nancy, les projets municipaux d'extension de la ville sont consignés dans le plan d'alignement de 1869, conçu juste avant le conflit de 1870, et qui ne sera pas revu avant le Plan Cornudet, plus de cinquante ans plus tard ${ }^{2}$. Il prévoit ainsi l'ouverture à l'urbanisation de nouveaux espaces, au-delà des faubourgs existants, par le biais de l'élargissement, du percement ou du prolongement de cinquante-six rues en grande partie nouvelles (Figure 1). Document de référence pour cibler les voies d'intérêt général, les priorités et orientations majeures à donner à la ville, il doit guider l'orientation de l'extension urbaine par la définition des secteurs à urbaniser en priorité. Mais non homologué par la Préfecture en raison de son inachèvement au moment du conflit de 1870, des libertés seront prises au fil des années avec la stricte application de ce plan, de moins en moins adapté à l'évolution enregistrée par la ville (Collot 1980).

En effet, l'afflux rapide et inattendu d'habitants et le développement de l'industrie livrent "la ville à quatre décennies de croissance effrénée » (Marseille, 2016), auxquelles le plan de 
1869 ne pouvait offrir de réponse suffisante. Par ailleurs, les moyens financiers de la ville ne peuvent suivre le rythme soutenu des ouvertures de rues à l'urbanisation, nécessaires pour accueillir l'ensemble des constructions nouvelles. De nombreuses initiatives privées couvrent alors les périphéries de rues et ruelles souvent sans grande cohérence, loin des préconisations du plan d'alignement. Face aux demandes d'ouverture ou de régularisation de ces voies privées, les municipalités successives sont amenées à hiérarchiser leurs projets et à définir les secteurs à conforter en priorité, s'éloignant souvent du plan d'alignement (Collot 1980, Dion 1983). Cette évolution de la politique de croissance urbaine ouvre de nouvelles perspectives au rôle potentiellement joué par le tramway.

Si le programme d'urbanisation des faubourgs développé dans le plan d'alignement de 1869 ne pouvait prendre en compte l'essor du tramway, qui lui est ultérieur, les nouvelles orientations des projets municipaux étaient en mesure d'orienter la croissance autour des axes naissants, aux tracés validés par le Conseil municipal. Le secteur spéculatif, qui organisait la croissance par le jeu des rues privées, aurait pu également s'avérer sensible à l'idée d'une plus-value générée par une offre de transport de qualité. On peut également poser l'hypothèse d'un rôle attractif joué par le tramway sur les habitants eux-mêmes : le gain de mobilité a pu être à même de favoriser l'attractivité, donc la valeur et le développement des quartiers longeant les axes desservis, préfigurant l'effet aujourd'hui attribué aux réseaux de tramway (Fritsch 2007).

Pour évaluer la véracité de ces impacts, il faut analyser finement la constitution de la ville au rythme de l'avancée du tramway. Les statistiques nous renseignent sur les secteurs qui absorbent plus ou moins efficacement les afflux de population (Tableau 2), mais ces derniers apparaissent très vastes et, sur les extérieurs, mêlent des espaces déjà urbanisés à de vastes zones encore vides. Par ailleurs, ces données ne fournissent pas la localisation précise des nouveaux bâtiments qui sortent de terre. Elles n'éclairent donc qu'imparfaitement l'évolution de la forme urbaine et l'impact éventuel des infrastructures de transport naissantes, et appellent au développement d'autres outils pour en affiner l'étude. L'objectif d'une analyse de l'adhérence ou l'indifférence du bâti nouveau au réseau de tramway a conduit à l'émergence d'un SIG historique, en cours de construction.

\section{B- L'apport de la démarche géohistorique appliquée à Nancy et au tramway}

18 La démarche géohistorique, "extension du champ de la géographie aux espaces du passé » (Valette et Carozza 2010) est entrée dans une nouvelle dimension grâce au SIG historique, véritable outil « d'histoire spatiale » (Gregory et Knowles, 2011). Cet outil, qui ne remplace pas mais accompagne recherches et analyses, permet en effet de capitaliser et de spatialiser des données du passé (Pons 2008).

L'appréhension des évolutions spatiales et temporelles d'un champ d'étude aussi mouvant et en constante évolution que le tissu urbain nous confronte en effet à plusieurs difficultés. Récentes ou historiques, les sources d'informations sont de natures diverses, et doivent être multipliées et croisées pour fournir l'information la plus exhaustive et précise possible. Le corpus documentaire constitué regroupe ainsi des sources d'archives, des cartes et plans anciens, des photographies aériennes ou au sol, des plans techniques ... répondant à des époques et à des échelles variées. Certaines de ces sources sont par 
nature spatialisées, à l'image des cartes ou des plans ; d'autres, notamment textuelles ou iconographiques, semblent ne pas se prêter spontanément à la représentation cartographique. Après un examen critique des sources, visant à ne retenir que celles qui offrent fiabilité et précision satisfaisantes, le SIG historique rend possible la visualisation et la superposition de ces informations d'époques et de natures différentes, et leur association dans le cadre d'études comparatives. Le géoréférencement des cartes et plans les replace à une échelle identique, facilitant leur comparaison, tandis que le remplissage des tables associées rend possible la spatialisation de documents textuels, qui ne semblaient pas a priori cartographiables. Outil d'origine quantitative, le SIG est ainsi à même de gérer et d'intégrer des sources de données qualitatives écrites, élargissant les domaines de recherche et les possibilités offertes à ses utilisateurs. Il rend possible la confrontation entre eux des documents anciens et contemporains à différentes échelles, présentant des différences géométriques et thématiques notables (Franchomme et Dubois 2010).

20 L'objectif de l'outil est ensuite double, à commencer par la production d'un recueil de cartes historiques, représentant l'évolution du tissu urbain et du réseau de transport à dates régulières. Mais l'outil SIG prend toute sa dimension dans l'exploitation des renseignements des tables associées aux éléments cartographiés. L'analyse spatiale offre une approche quantitative agrégée à la lecture spatiale des espaces cartographiés. Audelà de simples calculs et informations (superficies, densités, répartition de populations ou d'activités ...), l'outil facilite la mise en œuvre et la représentation cartographique d'analyses plus pointues.

21 Dans le cadre de notre étude sur Nancy, le SIG historique en cours de réalisation se concentre sur la reconstitution de l'évolution de la ville à l'échelle du bâtiment, suivant les pas de temps les plus précis possibles - un maximum de dix années a été retenu, mais il peut s'avérer plus court selon les documents récolés et les besoins de l'étude. Le corpus documentaire constitué regroupe deux grandes catégories de documents.

D'une part un ensemble de cartes et plans du XIXème et du XXème siècle - ont été retenus ceux présentant un niveau de finesse suffisant pour que le bâti soit représenté, tout en englobant l'intégralité de la ville de Nancy, voire quelques territoires alentours. L'intérêt de ces documents est de proposer d'emblée une spatialisation de l'avancée et de la transformation de la ville, bâtiment par bâtiment, sur des pas de temps réduits. Le croisement avec l'évolution du réseau de tramway s'en trouve simplifié. L'apport du SIG historique réside dans la possibilité de superposer ces éléments cartographiques, une fois dûment géoréférencés, favorisant alors leur rapprochement. Appuyée sur un fond cadastral actuel de près de 100000 polygones, la comparaison de plusieurs époques successives met en évidence l'évolution de la forme urbaine, la visualisation des nouvelles constructions, des bâtiments disparus ou remplacés. En mettant en perspective l'évolution des secteurs desservis par le tramway historique avec celle du reste de la ville, il est alors possible d'observer et de calculer la plus ou moins grande adhérence de l'urbanisation naissante à ces liaisons, et leur impact dans la constitution de la forme urbaine peut alors être approché (Figure 3). Cette étape, aux résultats déjà évocateurs, peut toutefois être affinée par l'apport de documents complémentaires. Mais manque de précision, erreurs du cartographe, datations hasardeuses, informations incomplètes ... les documents cartographiques ne peuvent se suffire à eux-mêmes.

Le recours à divers documents d'archive apporte d'autre part des informations à même de compléter les cartes et plans. Les permis de construire ainsi que le casier sanitaire des 
immeubles, généralisé à partir de la loi de $1902^{3}$, permet de définir avec assurance et précision la date de construction des immeubles, d'en connaitre l'élévation - et de s'assurer que l'immeuble d'origine est, ou non, toujours en place. La localisation des bâtiments industriels s'avère également un complément indispensable - leur présence participe de la compréhension de l'évolution de la ville et de ses transports, ainsi que de la localisation privilégiée des habitants dans certaines rues. Les tables nominatives des recensements, accessibles entre 1872 et 1936 peuvent y être associées, mais non sans qu'un important travail de dépouillement ait été préalablement réalisé. Elles apportent, entre autres exemples, des connaissances précises sur la densité de population à l'échelle du logement et du bâtiment, ou encore sur les professions exercées par les habitants. L'identification délicate de l'immeuble recensé sur un plan géoréférencé de date identique permet ainsi, par le remplissage de la table attributaire, la spatialisation et la représentation précise de ces informations qualitatives.

Figure 2

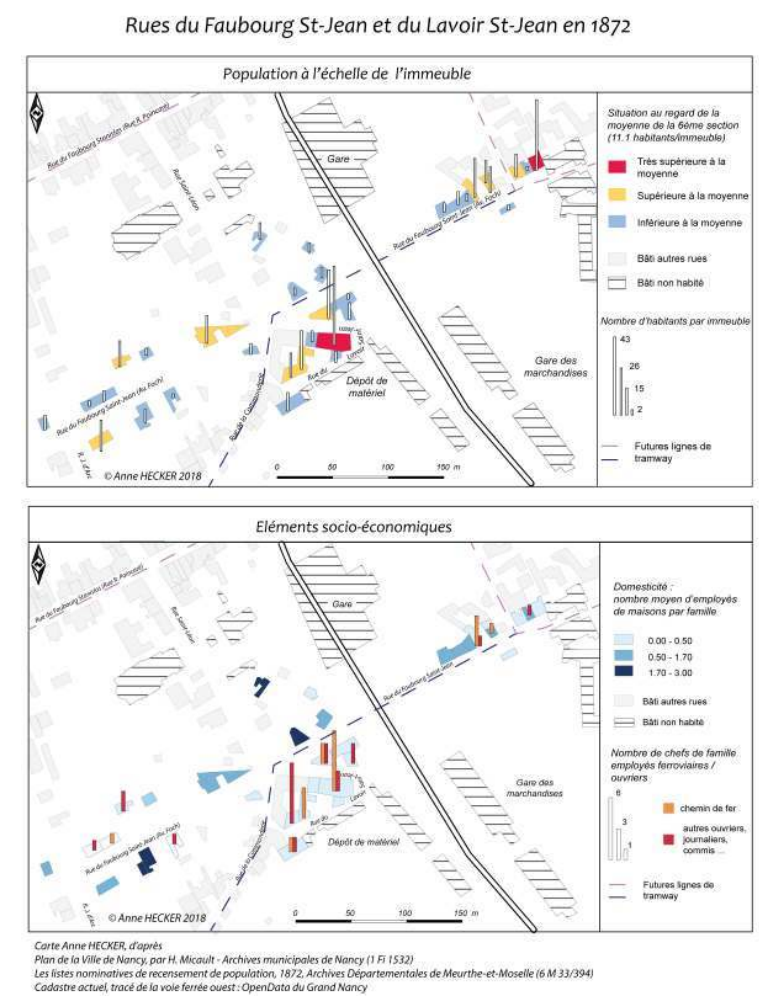

On peut ainsi s'arrêter sur l'exemple de deux rues contigües du front d'urbanisation occidental (sixième section), présentant dès 1871 de réelles différences. La représentation spatialisée des informations issues des tables nominatives du recensement de 1871 en favorise la comparaison (Figure 2). Ainsi, la petite rue du Lavoir St-Jean ne compte que sept bâtiments en 1871, dont une maison individuelle de six personnes mais surtout des immeubles, abritant jusqu'à quarante-trois habitants sous le même toit. La concentration de population y apparaît forte, globalement supérieure à la moyenne de la sixième section, de l'ordre de 11,1 habitants par immeuble. Les professions des trente-quatre chefs de famille trahissent la proximité des installations de la gare, à une date où le transport collectif est encore inexistant : $32.3 \%$ d'employés de chemins de fer, $35.3 \%$ d'ouvriers et journaliers (dont une partie travaille potentiellement également pour la compagnie de chemin de fer) et $17.6 \%$ d'artisans. Cette rue aux densités élevées présente 
donc un caractère ouvrier assez marqué, dans une section réputée pour avoir davantage concentré les constructions bourgeoises.

La rue contigüe du Faubourg St Jean s'avère plus disparate. Agrandie seulement depuis 1866 à l'ouest de la rue Saint-Léon, on y dénombre alors vingt-six immeubles. Si deux immeubles s'avèrent particulièrement peuplés (vingt-six et trente-huit habitants), dixhuit demeurent inférieurs à la moyenne de la section, et onze ne comptent que de petites cellules familiales de deux à cinq personnes. L'emploi ferroviaire et ouvrier y est représenté, mais bien plus faiblement (respectivement sept et huit chefs de famille sur soixante-dix-huit), et se localise majoritairement à l'angle de la rue précédente ou dans la partie à l'est de la voie ferrée, à proximité de la gare. La petite bourgeoisie représente près de $50 \%$ des familles, essentiellement concentrées dans cette partie orientale, la plus proche du centre-ville et la plus densément habitée. A l'ouest de la voie ferrée, sur le front d'urbanisation, les maisons se font moins proches, se composent davantage de structures mono-familiales, parfois complétées de la location d'un logement. La grande bourgeoisie, employant une domesticité plus importante, y est davantage représentée. En l'espace d'un tour de rue, deux mondes différents s'apprêtent à accueillir le tramway.

La répétition de ce travail de représentation des données d'archive sur le temps long, au fil des périodes censitaires, permet de voir évoluer ces quartiers, d'apporter des éléments de réponse éclairant l'impact des lignes de tramway historiques sur la répartition sociospatiale des habitants dans la ville, et d'évoquer des trajectoires urbaines, sociales et humaines de ces espaces.

\section{II- L'impact du tramway historique sur la ville}

27 L'analyse que favorisent les cartes produites à l'aide du SIG historique nous éclaire sur cette double question de l'intégration des lignes historiques dans leur environnement urbain : entre desserte adhérente à l'espace déjà densifié, et infrastructure pionnière dans des étendues encore en marge de la ville, quel rôle endossent les liaisons de tramway historique à Nancy? Ont-elles notamment pu, à une échelle fine, participer à modifier densité et caractères socio-économiques à leur abord immédiat? Et une fois établies, sont-elles devenues des liaisons structurantes participant à composer la forme urbaine, à guider l'urbanisation et concentrant population et activités autour d'elles?

\section{A- Entre adhérence à l'urbanisation préexistante et caractère pionnier}

28 À l'instar des lignes de transport collectif « lourdes » actuelles, le réseau historique ne peut se soustraire à la desserte du centre ancien - en 1873, à la veille de l'arrivée de la première ligne, ce dernier représente en effet 50 \% de la surface bâtie, $57 \%$ de la population de Nancy et de nombreux activités et pôles générateurs de trafic. Sa desserte semble donc inévitable. La première ligne (Figure 1) irrigue ainsi les villes Neuve de Charles III et de Stanislas, aux trames viaires larges et régulières, puis frôle par l'ouest la ville médiévale au nord, aux rues étroites et surpeuplées, peu propices à son passage (Bastien 2003). Par la suite, les deux lignes qui complètent le premier réseau du XIXème siècle renforcent la desserte de la ville-centre (Figure 3), en doublant la première ligne par l'est, et en assurant, via une grande artère commerçante, la desserte est-ouest. Son intersection avec la précédente forme l'amorce du «Point Central », futur carrefour et 
centre de correspondance des transports collectifs nancéiens. Les lignes du XXème siècles viendront ensuite s'articuler sur ces premiers itinéraires centraux, et assurer la desserte des quartiers extérieurs ${ }^{4}$.

Figure 3

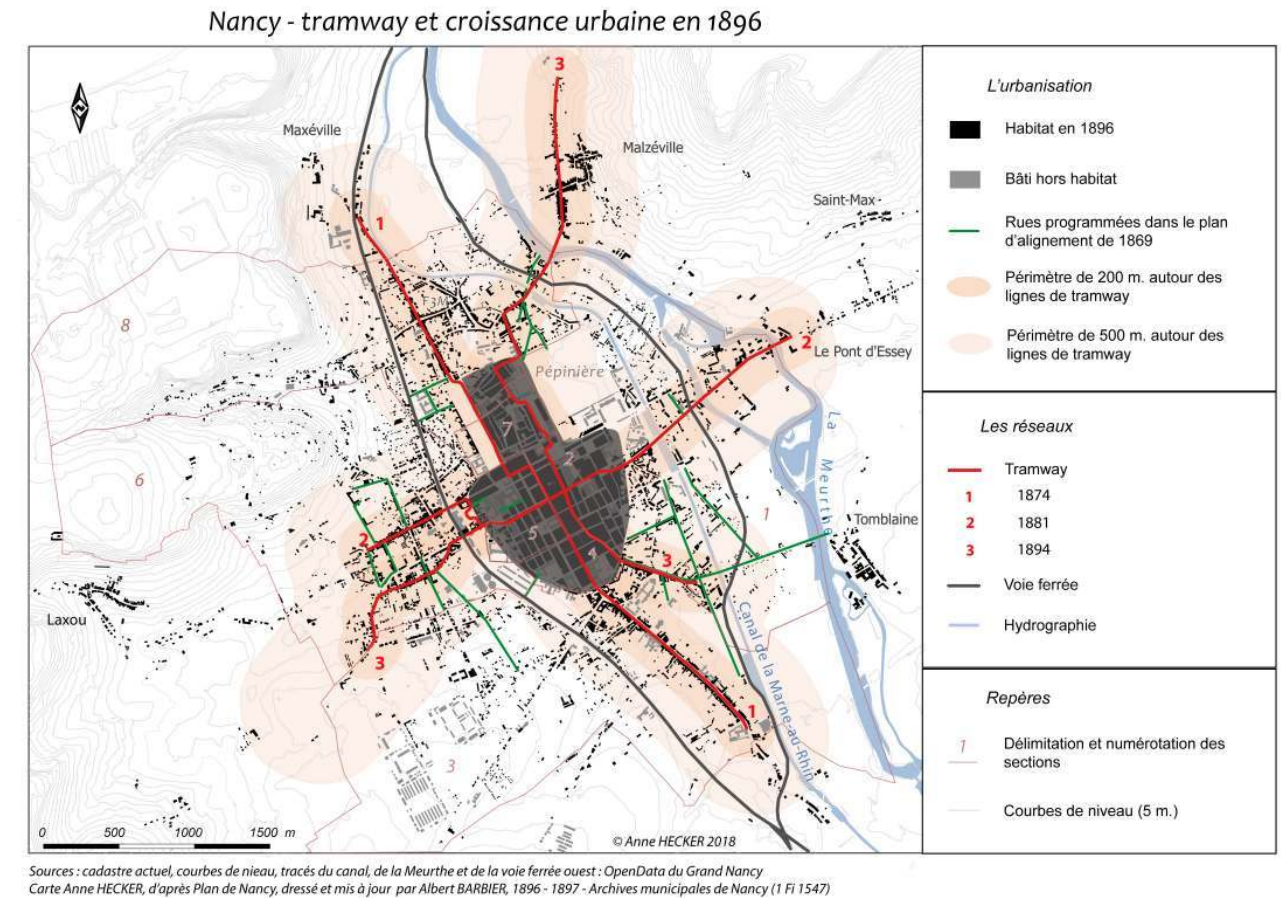

$\mathrm{Au}$ sortir de la ville-centre, les nouvelles lignes de tramway desservent, au moment de leur inauguration, des quartiers d'autant moins densément peuplés que la mise en service est précoce - la ville s'écarte progressivement de ses limites originelles, et aux immeubles urbains se mêlent des maisons individuelles avec jardin, favorisant une urbanisation moins dense. Le tracé de la ligne $1^{5}$ illustre assez bien le choix qui s'offre aux concessionnaires de ces liaisons. En effet, les espaces en croissance dans les sections extérieures alternent des zones quasi-vides, ponctuées d'habitat et d'activités éparpillées, et des faubourgs qui s'étirent depuis les portes de la ville, et qui concentrent une partie des habitations excentrées. La première ligne emprunte ainsi le long Faubourg SaintPierre au sud, et les suivantes desserviront Saint-Jean et Saint-Stanislas à l'ouest, et Sainte-Catherine à l'est. En revanche, la ligne 1 s'écarte au nord du Faubourg des TroisMaisons. À sa traversée a été préféré un itinéraire parallèle plus à l'ouest, et dont la section terminale rejoint les bornes de la ville à travers un espace très peu peuplé. À la logique de la desserte d'une population agglomérée se substitue alors celle de la mise en relation de la ville avec un espace productif, celui des Brasseries de Maxéville. Cette même logique guidera également la seconde ligne vers l'industrie naissante entre Meurthe et canal en direction de Pont d'Essey, et la troisième ligne au-delà du village de Malzéville, lui-même en partie évité (Figure 3) (Hecker 2018).

On peut voir dans ces itinéraires de tramway une certaine adhérence à l'existant faubourgs, activités économiques - mais également un aspect pionnier. En effet, la population agglomérée, même au long des faubourgs, demeure peu nombreuse - en témoigne la faible épaisseur de l'espace urbain qui encadre la première ligne dans le faubourg Saint-Pierre. Les segments septentrionaux des lignes 1 et 3 traversent quant à 
eux, au moment de leur ouverture, des espaces encore moins densément habités, voire quasi-vides à l'approche du terminus. Même les extensions plus tardives du début XXème ou des années 1930 s'écarteront à nouveau de la ville densifiée pour s'implanter sur le front urbain, encore peu densément construit. Il serait donc possible d'évoquer des lignes pionnières - cela sous-entendrait toutefois qu'une fois implantées, elles deviendraient sources de structuration de l'espace en cours d'urbanisation, attirant population et activités dans leur sillage. Deux causes pourraient alors avoir favorisé une telle attractivité : celle spontanée et privilégiée de la population et des activités, attirées par les atouts d'une mobilité favorisée ; et / ou une orientation de celles-ci vers les axes bien desservis, favorisée par les municipalités successives. Dans cette optique, une cohérence entre le plan d'alignement et le tracé des lignes de tramway concédées, l'ensemble étant validé par le Conseil Municipal, serait logiquement attendu. Or on constate, notamment sur la Figure 3, l'imparfaite cohérence du tracé des rues à ouvrir à l'urbanisation, et des itinéraires des lignes de tramway.

31 Avant le conflit de 1870 et l'afflux massif de population qui allait suivre, le plan d'alignement de 1869 oriente l'extension de la ville hors de ses murs. Il prolonge les extensions programmées dans le plan d'alignement précédant de 1846. Le projet de rues nouvelles à ouvrir à l'urbanisation (Figure 1) accompagne un premier développement de l'habitat, notamment bourgeois, vers l'ouest de la ville, hors d'eau et des nuisances de l'industrie naissante à l'est. Plusieurs rues y sont programmées, globalement perpendiculaires aux deux faubourgs préexistants. Ils étirent la ville dans le sens nordouest - sud-est, alors dominant, et souligné par la Meurthe et les diverses infrastructures de transport. À l'opposé, les grands espaces de fond de vallée de l'est, demeurés vacants en raison des risques d'inondation (Chiffre et Mathis 2014), disponibles, plats, proches du canal, puis après 1881 de la gare de marchandises, sont destinés dès 1869 à cette activité industrielle que la ville déverse vers ses périphéries les moins attractives. On y prévoit quelques longues rues à même de structurer cet espace encore vide. Enfin, un troisième secteur, entre le faubourg des Trois Maisons, la vieille ville et le pont historique de Malzéville, profite de la libération des glacis pour faire émerger le projet d'un petit réseau de rues à construire.

Un dernier ensemble de rues à ouvrir à l'urbanisation ceinture la nouvelle Manufacture des Tabacs, à l'est de la voie ferrée. Ce sera le seul ensemble à être desservi par la première ligne de tramway, qui reste sinon à l'écart des projets municipaux. En revanche, les segments de lignes de tramway qui ouvrent à l'ouest (1881 et 1894) desservent les deux faubourgs sur lesquels s'articulent les rues nouvelles à créer. Le réseau de tramway semble ainsi gagner en cohérence avec le projet municipal, de même que pour la ligne Malzéville - Lobau (Ligne 3, à l'est). Mais entre-temps, l'urbanisation s'est développée et s'est largement affranchie du projet de 1869. Confrontées à l'urgence d'une croissance de population très rapide, les municipalités transigent et acceptent les projets d'initiative privée, qui contournent le plan d'alignement de 1869 et écartent la croissance urbaine des axes initialement prévus (Hecker 2018, Collot 1980).

\section{B Des lignes attractives?}

33 La cohérence des constructions nouvelles se détache donc des projets municipaux. On pourrait alors envisager que c'est pour mieux s'attacher au réseau de tramway naissant. Mais pourtant, elles n'adhérent que partiellement à ce dernier. Ainsi, la spatialisation des 
constructions en 1896 illustre une croissance urbaine quasi ubiquiste (figure 3). En isolant le bâti concentré dans un rayon de $200 \mathrm{~m}$ autour des premières lignes de tramway, on perçoit mieux l'impact relatif du tramway sur la localisation de l'urbanisation nouvelle ${ }^{6}$. Le tableau 3 isole les segments de ligne au sortir du centre historique : au cœur de ce dernier le bâti, originellement très dense, a peu évolué quant à son emprise au sol. Quelques rues y sont remaniées, des bâtiments remplacés, mais à quelques notables exceptions près, ces modifications n'entrent pas dans le champ de notre étude, offrant au centre une image de relative stabilité quant à la consommation d'espace dédié au bâti. Nous nous intéresserons donc aux seuls segments de ligne s'implantant dans les sections extérieures de la ville, alors en pleine évolution.

Tableau 3 : Superficies bâties autour des premières lignes de tramway

\begin{tabular}{|c|c|c|c|c|c|c|c|}
\hline \multicolumn{3}{|l|}{ Tramway } & \multicolumn{3}{|c|}{$\begin{array}{l}\text { Emprises bâties dans la zone tampon } \\
\text { de } 200 \mathrm{~m} \text {. autour de la ligne de } \\
\text { tramway }\end{array}$} & & \multirow[b]{2}{*}{$\begin{array}{l}\text { Durée mise } \\
\text { en service } \\
1896\end{array}$} \\
\hline Section & $\begin{array}{l}\text { Mise en } \\
\text { service }\end{array}$ & Longueur & 1874 & 1885 & 1896 & $\begin{array}{ll}1896: \\
\text { ha } & \text { bâtis } \\
/ & \mathrm{km} \\
\text { tram } & \end{array}$ & \\
\hline \multirow{2}{*}{ S1 Nord } & \multirow{2}{*}{1874} & \multirow{2}{*}{$1,9 \mathrm{~km}$} & $5,6 \mathrm{ha}$ & 11,7 ha & 13,5 ha & \multirow{2}{*}{7} & \multirow{4}{*}{22 ans } \\
\hline & & & $5,40 \%$ & $7,60 \%$ & $7,50 \%$ & & \\
\hline \multirow{2}{*}{$\begin{array}{l}\text { S1 Sud } \\
\text { F. St-Pierre }\end{array}$} & \multirow{2}{*}{1874} & \multirow{2}{*}{$1,4 \mathrm{~km}$} & 8,5 ha & $11,02 \mathrm{ha}$ & 13,2 ha & \multirow{2}{*}{9,9} & \\
\hline & & & $8,20 \%$ & $7,20 \%$ & $7,30 \%$ & & \\
\hline \multirow{2}{*}{$\begin{array}{l}\text { S2 Ouest } \\
\text { F. St- } \\
\text { Stanislas }\end{array}$} & \multirow[b]{2}{*}{1881} & \multirow[b]{2}{*}{$1,2 \mathrm{~km}$} & 3,7 ha & 9,8 ha & 11,8 ha & \multirow[b]{2}{*}{10} & \multirow{4}{*}{15 ans } \\
\hline & & & $3,60 \%$ & $6,40 \%$ & $6,60 \%$ & & \\
\hline \multirow{2}{*}{$\begin{array}{l}\text { S2 Est } \\
\text { F. Ste- } \\
\text { Catherine }\end{array}$} & \multirow[b]{2}{*}{1881} & \multirow[b]{2}{*}{$1,5 \mathrm{~km}$} & 4,9 ha & 6,2 ha & $7,8 \mathrm{ha}$ & \multirow[b]{2}{*}{5,3} & \\
\hline & & & $4,70 \%$ & $4 \%$ & $4,30 \%$ & & \\
\hline \multirow{2}{*}{$\begin{array}{l}\text { S3 Nord } \\
\text { Malzéville }\end{array}$} & \multirow{2}{*}{1894} & \multirow{2}{*}{$2,1 \mathrm{~km}$} & 4,9 ha & 8,7 ha & 10,1 & \multirow{2}{*}{4,9} & \multirow[t]{4}{*}{2 ans } \\
\hline & & & $4,70 \%$ & $5,70 \%$ & $5,60 \%$ & & \\
\hline \multirow[t]{2}{*}{ S3 Sud } & \multirow[t]{2}{*}{1894} & \multirow[t]{2}{*}{$0,5 \mathrm{~km}$} & $1,4 \mathrm{ha}$ & 3,2 ha & $\begin{array}{l}5,1 \text { ha (dont } 1,4 \\
\text { ha commun avec } \\
\text { L1 Sud) }\end{array}$ & \multirow[t]{2}{*}{9,4} & \\
\hline & & & $1,30 \%$ & $2,10 \%$ & $\begin{array}{l}2,8 \quad \% \quad(1,8 \% \\
\text { commun })\end{array}$ & & \\
\hline
\end{tabular}




\begin{tabular}{|l|l|l|l|l|l|l|}
\hline \multirow{2}{*}{$\begin{array}{l}\text { S3 Ouest } \\
\text { F. St-Jean }\end{array}$} & \multirow{2}{*}{1894} & 2,1 ha & 9,5 ha & $\begin{array}{l}10,4 \text { ha (dont } 5,4 \\
\text { ha commun avec } \\
\text { L2 Ouest) }\end{array}$ & 8,7 \\
\cline { 3 - 6 } & $2 \%$ & $6,20 \%$ & $\begin{array}{l}5,8 \% \\
\text { commun) }\end{array}$ & \\
\hline
\end{tabular}

NB les zones en grisé représentent les emprises avant la mise en place du tramway Les pourcentages représentent la part des surfaces bâties dans la zone tampon, rapportées à l'ensemble des surfaces construites à la même date (Nancy et proches villages desservis) Les numéros des segments, établis dans l'ordre de création des lignes, sont identiques à ceux employés sur la Figure 3

Sources - données de l'auteur - QGis

L'analyse des corridors de $200 \mathrm{~m}$. autour des lignes de tramway nous permet de tirer cinq grands constats quant à l'impact de la présence de ces infrastructures sur la densité du bâti :

Même pionnières, les lignes de tramway ne s'implantent pas dans des espaces «vides ». Les zones en grisé l'illustrent, de 2 à $6 \%$ du bâti de la ville est préexistant dans le périmètre qui s'apprête à accueillir l'une de ces lignes ;

- Une fois établies, les lignes ne présentent pas un caractère répulsif, la construction s'y poursuit, à l'image du reste de la ville - tous les corridors enregistrent l'apparition de nouveaux bâtiments. Ainsi entre 1874 et 1896, les superficies construites, exprimées en hectares, s'accroissent de $141 \%$ au nord de la ligne 1, et de $55 \%$ pour la partie sud ;

- Bien que non répulsifs, ces périmètres n'apparaissent pas pour autant particulièrement attractifs. Certes, la construction s'y poursuit, mais la croissance est également forte dans les secteurs de la ville non desservis. Ainsi, si l'on se concentre sur les seules constructions nouvelles, on constate qu'entre 1873 et 1885 , la ville s'accroît de 40 ha de bâti. Le périmètre de la ligne 1 ne concentre alors que $17 \%$ de ces constructions nouvelles $(6,9 \mathrm{ha})$, à peine plus que la ligne $2(6,1$ ha, $15 \%)$ - soit, à elles deux, moins d'un tiers de la superficie nouvellement bâtie dans la ville. La Figure 3 montre l'émiettement du bâti sur l'essentiel du périmètre urbain, à l'exception des zones marquées par de fortes pentes et sur le sommet du plateau à l'ouest. Le tableau confirme cette observation. En dépit d'un fort accroissement des espaces bâtis le long du segment nord (+141\%), il ne représente en 1896 que $7.5 \%$ du bâti urbain, seulement deux points de plus que 22 ans auparavant. Si la croissance absolue est partout marquée, l'évolution relative est quant à elle souvent modeste, voire négative, traduisant une croissance plus forte dans le reste de l'agglomération que le long de ces segments de tramway ;

- Le temps ne fait rien à l'affaire, au moins jusqu'en 1896. La croissance est, à l'image de l'évolution générale de la ville, très forte entre 1873 et 1885, puis se ralentit, ce qui se traduit tant dans les données absolues que relatives. Ainsi, même après 22 ans d'implantation, la ligne 1 ne concentre pas plus de 7.3 à $7.5 \%$ du bâti urbain, en dépit de vastes espaces encore disponibles autour des deux segments nord et sud. Quant à la deuxième ligne, les onze années qui la séparent de sa mise en service n'ont permis à ses deux segments de ne gagner que 0.3 et 0.2 points de bâti en plus ;

- Enfin, la localisation des lignes de tramway dans la ville possède un impact sur la plus ou moins forte adhérence de l'urbanisation nouvelle à la ligne. Les corridors situés au nord et à l'est totalisent moins d'hectares bâtis par kilomètre (entre 4.9 et 7) que le sud et l'ouest (autour de 10) - indépendamment du temps depuis lequel la ligne est en service. 
36

argissement à un périmètre de $500 \mathrm{~m}$. autour des voies (voir Figure 3 et note 6 ) renforce certains de ces constats. Ainsi, les extrémités, particulièrement au Nord et à l'Est, ne comptent guère plus de constructions à desservir au-delà des deux cents premiers mètres, contrairement aux espaces plus proches du centre, ou localisés à l'ouest ou au sud de la ville. Si une part essentielle de l'espace urbanisé peut être considéré comme potentiellement desservi par le tramway car situé dans un périmètre accessible en une dizaine de minutes de marche, des espaces demeurent hors de sa portée, dessinant des «zones blanches» en cours d'urbanisation, encore mal desservies. Elles laissent deviner les futurs tracés des lignes qui viendront compléter le réseau au XXème siècle.

Ainsi, sur Nancy, ni les premières municipalités, ni les spéculateurs et entrepreneurs privés ne semblent avoir réellement considéré le tramway comme un guide puissant, pouvant être mobilisé pour conduire, ou simplement valoriser, les projets de développement urbain. Mais peut-on voir un impact sur une répartition plus fine de la population? L'analyse des tables nominatives nous éclaire sur l'évolution des densités de population, et non uniquement sur celle des constructions, ainsi que sur les modifications socio-économiques des secteurs étudiés.

Ainsi, nos deux rues évoquées dans la première partie se localisent à un peu plus de $200 \mathrm{~m}$. de la ligne 2, ouverte en 1881 , et au cœur du corridor de la troisième ligne (segment ouest; 1894). La croissance de la rue du Faubourg-Saint-Jean est sensible, le nombre d'immeubles croît de 127 \% entre 1874 et 1896, mais surtout, le nombre d'habitants et de familles triple dans le même pas de temps. Pourtant $50 \%$ des nouvelles constructions demeurent à un ou deux logements, impliquant une densité renforcée dans le reste des immeubles. Grande bourgeoisie (28\%) et employés (33 \%) se renforcent, de même que la présence de la domesticité (116 domestiques déclarés au service de 232 familles), particulièrement dans la partie ouest de la rue. Quant à la petite rue du Lavoir Saint Jean, son évolution en nombre de bâtiments reste modérée (de 7 à 11) faute de place disponible. En revanche, le nombre de familles et d'habitants, déjà élevé, y double dans le même laps de temps. La densité se renforce à 20 habitants par immeuble en moyenne, contre 12 dans la rue adjacente. Le nombre de chefs de famille employés "au chemin de fer " reste stable, la proximité de l'emploi demeure encore importante malgré le déploiement du réseau de tram. En revanche, le nombre d'ouvriers se restreint (-40\%) au profit des artisans et des commerçants (+126\%). Ces deux rues traduisent ainsi l'évolution du quartier, où globalement, le prix des loyers s'élève dans les rues adjacentes (Dion 1980). La poursuite de l'étude devrait permettre d'inscrire l'évolution de ces rues dans un tableau plus général à l'échelle de la ville, et mettre au jour d'autres impacts des lignes de tramway que celui de la seule densité des constructions. 


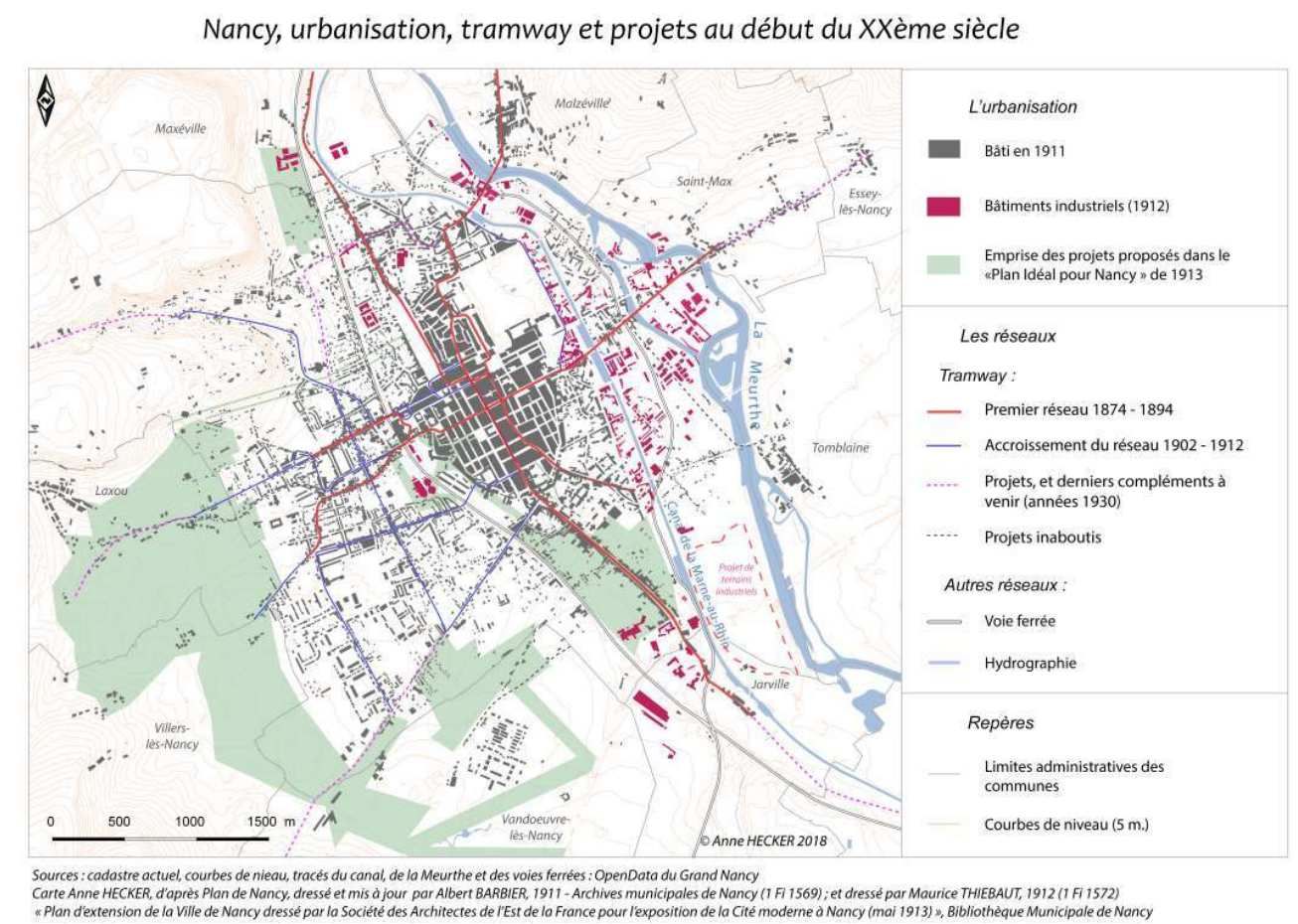

En effet, nous venons de souligner une adhérence mitigée des nouvelles constructions aux lignes de tramway, mais l'analyse laisse apparaître en filigrane d'autres cohérences, notamment celle liée à la localisation des lignes dans les différentes parties de l'espace urbain. Ainsi, la partie orientale de la ville n'accroît que lentement son bâti mais voit en revanche se multiplier d'autres pôles générateurs de trafic, particulièrement les établissements industriels (Figure 4). Ils se multiplient particulièrement autour du canal et de la Meurthe, espaces mal desservis par le tramway. Certains de ces établissements sont implantés dans le périmètre de la deuxième ligne de tramway reliant Pont d'Essey (usine Daum, usine à gaz, anciens abattoirs ...); d'autres seront raccordés à la ligne ajoutée au début du siècle longeant la Pépinière par l'ouest. Les implantations industrielles s'étirent toutefois prioritairement parallèlement aux infrastructures lourdes (canal, voie ferrée dédiée aux marchandises), et notamment progressivement vers le sudest, comme en témoigne le projet de nouveaux terrains industriels aux portes de Jarville (figure 4). Elles n'adhèrent donc que modestement à un réseau de tramway, qui ignore cette nouvelle direction majeure, et concentre son développement à l'ouest de la ville.

Ciblé pour grandir dès les premiers projets de 1869, l'Ouest enregistre à l'inverse une forte densification des constructions ainsi que des populations, notamment dans le périmètre des lignes de tramway, ainsi que l'illustre l'évolution de nos deux rues. Elle nous rappelle ainsi que «le tramway pas plus que le métro n'ont de "vertu" urbanistique propre. Les mutations doivent être préparées et maitrisées » (Guth, 2008). Les capacités structurantes des infrastructures ne peuvent s'affirmer que lorsque le territoire qui les accueille présente des opportunités de développement, portées par des projets élaborés en amont, " quand le projet précède l'effet spontané pour produire un effet programmé » (Beaucire 2014).

41 Certes les constructions réellement engagées se détachent des projets municipaux, mais à la fin du XIXème s., ce sont bien les sections 3, 6 et 8, à l'ouest de la voie ferrée, qui concentrent l'essentiel des nouvelles constructions, au détriment du nord et de l'est. C'est 
également dans cette partie occidentale que se localisent les principaux projets de tramway au cours des décennies à venir. Une seule ligne d'1,4 km, verra le jour à l'est du centre au début XXème (1902 - 1912), alors que dans le même temps, elles se multiplient dans la partie ouest, totalisant plus de $14 \mathrm{~km}$ (Figure 4).

Ce constat est renforcé par l'analyse de deux documents complémentaires, le « Plan idéal pour Nancy $»^{7}$, qui servira de base pour l'élaboration du «Plan d'aménagement, d'embellissement et d'extension » dit «Plan Cornudet », prescrit par la loi d'urbanisme de 1919, entamé en 1922 et approuvé en 1932. Ils ciblent les espaces encore disponibles dans la tache urbaine du début XXème siècle, et proposent de grandes orientations pour l'expansion urbaine à venir. Ils soulignent et prolongent encore la croissance remarquable de l'ouest de la ville, débordant sur les communes limitrophes. Ils démontrent également la faible adhérence déjà observée autour de la première ligne de tramway - deux projets de croissance urbaine s'inscrivent autour ou à proximité de son tracé, proposant de mobiliser les opportunités foncières qui demeurent disponibles, quarante ans après sa mise en service. Une extension de 55 ha englobe notamment l'extrémité méridionale du corridor, qui a conservé sa morphologie de faubourg à peine étoffée, pointant le faible usage qui a jusqu'alors été fait de cet espace, pourtant idéalement placé et sans grande contrainte topographique.

Mais cette nouvelle orientation pour la croissance de la ville, soixante ans après le plan d'alignement de 1869 , souligne également une évolution notable de la relation entre ville et tramway: tous les espaces désignés pour grandir se trouvent desservis par les lignes existantes, mobilisant six segments de lignes - quatre à l'ouest, et les deux segments nord et sud de la première ligne. En revanche, en dépit d'espaces encore disponibles dans ses limites administratives, les projets s'exportent massivement hors de Nancy, et s'implantent en bout de ligne - seul le projet au sud, précédemment évoqué, englobe l'infrastructure au cœur de l'enveloppe à urbaniser.

L'orientation méridienne originelle soulignée par son environnement (front de côte, cours de la Meurthe) et les voies de communication (route nationale, voie ferrée, canal, première ligne de tramway), laisse place à une extension transversale majoritaire pour l'habitat. En témoignent l'orientation principales des segments ouverts au début du XXème, et jusqu'aux derniers ajustements des années 1930, mais également l'abandon précoce des lignes vers Maxéville et Malzéville (au nord), pourtant relevant du premier réseau. Déficitaires, elles seront les premières à être remplacées par des services de bus. Le réseau de tramway historique sera ensuite peu à peu démantelé entre 1948 et 1958, laissant la ville sans réseau lourd durant une vingtaine d'années, uniquement desservie par un système d'autobus, dont les performances et l'attrait parmi la population décroissent rapidement.

\section{Conclusion - Quels apports à la connaissance du lien tramway / urbanisation?}

Depuis son retour dans les villes françaises il y a quarante ans, le tramway a donné naissance à de nombreuses publications abordant différents aspects, notamment celui du lien tissé avec l'espace urbain. On insiste ainsi sur le double rôle du tramway infrastructure de déplacement et outil de recomposition urbaine revalorisant l'espace public (Meunier-Chabert et Trêve-Thomas 2018, Laisney 2011) - outil au service d'une 
politique de marketing territorial, voire "référent principal pour l'identité des villes" (Redondo 2012). Désormais bien intégré dans la ville, son potentiel de guide de la croissance urbaine introduit l'idée d'une articulation, voire d'une subordination de l'urbanisation nouvelle à ce réseau, par le biais notamment de contrats d'axe ou autres DIVaT, tentatives de Transit Oriented Development à la française (Bentayou et al. 2015, Maulat 2015). Les chercheurs s'intéressent également à la relocalisation économique et commerciale au long des axes - qui n'enregistre pas toujours les effets escomptés (Meunier-Chabert et Trêve-Thomas 2018) - mais aussi aux effets indirects du tramway tels que l'augmentation des prix du foncier, ou son potentiel de gentrification (Fritsch 2007). Mais la littérature française ne s'intéresse que rarement à la co-construction de la ville et de son aîné, le tramway historique.

Bien souvent, son analyse se concentre davantage sur la description du réseau, de ses aspects matériels et techniques, et sur les dates clés de son histoire (Robert 1974), parfois simples paragraphes préalables à la description du réseau actuel (Laisney 2011, Tricoire 2007). Les mutations urbaines et sociales, « induites par la capacité de se déplacer " (Flonneau et Guigueno 2009) sont quant à elles rarement abordées. Les études se sont davantage penchées sur le rôle du rail, sa diffusion de la croissance urbaine en doigts de gant, sa participation à la dissociation habitat / travail (Merlin 1982, Schaeffer et Sclar 1980), et la naissance des quartiers de gares (Lambert-Bresson 2016, Bondon 2011). L'impact polarisant du tramway sur l'urbanisation est davantage mis en avant par la littérature américaine (Block-Schachter 2012, Brooks et Lutz 2016), qui s'intéresse à la singularité actuelle des Streetcar suburbs, quartiers aujourd'hui encore plus denses et aux mobilités plus vertueuses que les secteurs nés de l'automobile.

Que retenir de cette étude?

Ainsi en dépit d'une littérature abondante, on constate que le lien entre les transports publics urbains émergents fin XIXè siècle et la fabrique de la ville s'avère peu fréquemment abordé. Cette analyse offre ainsi un regard nouveau, dans l'objectif d'une possible modélisation de la capacité structurante des lignes de tramway, qui offrira des comparaisons possibles et instructives avec d'autres villes. On observe toutefois que les principaux apports de cette étude recoupent certaines observations relevant de l'inscription des lignes récentes de tramway dans la ville.

Ainsi, si « le rail a [...] la capacité d'accélérer l'organisation de l'espace, il possède des capacités structurantes et polarisantes, il accompagne, oriente, souligne les dynamiques démographiques et explique des contrastes durables dans la répartition des richesses " (Baron et Mussulan 2017). Certainement peut-on en dire autant, au moins partiellement, de cette autre forme de rail que constitue le tramway historique. La forte croissance démographique et industrielle, la rapide croissance de l'espace urbain sont des éléments de contextes indispensables, qui ont nourri l'extension du réseau de tramway - et ce dernier a participé à organiser la ville.

Mais déjà au XIXème siècle, à l'instar d'aujourd'hui, le contexte géopolitique local, le poids des décisions et des négociations (Hamman 2018), la question foncière ... s'avèrent des points fondamentaux pour la compréhension des tracés, mais aussi de l'adhérence ou de l'indifférence urbaine qui s'y rattache. A l'instar de quelques bilans qui démontrent aujourd'hui que "les effets du tramway sur le territoire ne sont pas systématiques " (Meunier-Chabert et Trêve-Thomas 2018), toutes les lignes de tramway historique n'ont pas su guider l'urbanisation et les implantations économiques. Le parallèle peut 
également être fait avec l'urbanisation des secteurs de gares, lents et n'aboutissant que grâce à une politique volontariste émanant des municipalités, au gré des pressions foncières et démographiques (Bondon 2011) - une variable également très lisible dans le cas du tramway historique.

Facteurs de cohésion et de densification des secteurs péricentraux, ou "vecteurs potentiels du processus de délitement des territoires [...] favorisant l'éclatement des fonctions urbaines et le processus d'étalement urbain " (Gallez et Chardonnel 2018), le rôle du tramway dans la constitution de la ville présente des ambivalences. L'impact socio-économique à échelle fine, qui semble amorcer un processus de zoning voire de gentrification avant l'heure, reste également à approfondir, mais semble une approche prometteuse. De même, la localisation fine d'autres équipements générateurs de trafic et de polarisation des quartiers, celle des équipements commerciaux notamment, pourrait s'avérer éloquente. A la suite de Keeney (2017), peut-être démontreront-ils de nouvelles logiques d'implantation de la population, à court et à long terme, participant à expliquer un autre facteur observé dans le cas de Nancy, déjà étudié en région parisienne (Desjardins et al.2012) mais qui reste à explorer ici, celui de la permanence dans le temps long des itinéraires, même longtemps après que l'infrastructure ait disparue.

\section{BIBLIOGRAPHIE}

Baron N., Messulam P., 2017, Réseaux ferrés et territoires : La géographie humaine du chemin de fer. Un retour aux sources, Paris, Presses de l'école nationale des Ponts et Chaussées, 368 p.

Bastien P., 2003, Évolution des transports en commun urbains de 1820 à nos jours, Thèse sous la direction de Sylvain SCHIRMANN, soutenue à l'Université de Metz, U.F.R. Sciences humaines et Arts, 2 tomes, $1291 \mathrm{p}$.

Beaucire F., 2014, « De l'effet structurant au projet structuré », in « Les effets structurants des infrastructures de transport ", L'Espace géographique, 1/2014 (Tome 43), p. 51-67.

Bentayou G., Perrin E. et Richer C., 2015, « Contrat d'axe et Transit-Oriented Development : quel renouvellement de l'action publique en matière de mobilité et d'aménagement? (Point de vue d'acteurs) ». Flux, n 101-102, p. 111-123

Block-Schachter D., 2012, Hysteresis and urban rail : the effects of past urban rail on current residential and travel choices, Thèse, Massachusetts Institute of Technology, $284 \mathrm{p}$.

Bondon A., 2011, "Chemin de fer et forme urbaine au XIXème siècle ", in Lambert-Bresson M. et Térade A., 2011, Architectures urbaines et architectures du mouvement 1800 - 1950, Paris, Editions Recherches / IPRAUS, $180 \mathrm{p}$.

Burchfield M. et al., 2006, "Causes of Sprawl: A Portrait from Space”, The Quarterly Journal of Economics, $\mathrm{n}^{\circ} 121.2$, p. 587-633.

Brooks L., and Lutz B., 2013, Vestiges of Transit: Urban Persistence at a Micro Scale. 74p. (document de travail) 
Calvayrac M., 1913, « L'extension des villes et les tramways », Bulletin de la Société industrielle de l'Est, $n^{\circ} 113$, octobre 1913, p.6-33

Chiffre E., Mathis D., et Mathis A., 2014, « Les inondations à Nancy - Anciennes et nouvelles problématiques ». Développement durable et territoires. Économie, géographie, politique, droit, sociologie, Vol. 5, n³ -2014, 19p.

Collot C., 1980, « Les politiques municipales d'urbanisme à Nancy de 1871 à 1914 », in Études d'histoire municipale à la mémoire de Claude Collot, L'urbanisation de Nancy entre 1871 et 1914 Nancy, Publications de l'Université Nancy II, p. 5 - 128

Delpirou A., 2009, La fin de la ville loin du fer ? Transport et urbanisation dans la Rome contemporaine : les politiques publiques face aux héritages territoriaux, Thèse de Doctorat de Géographie, sous la direction de Colette VALLAT, soutenue le 27 novembre 2009, Université Paris 10 Ouest Nanterre La Défense, $643 \mathrm{p}$.

Desjardins X., Douay N., Gagnière V., 2012, «Évolution des schémas, permanence des tracés : la planification de la région parisienne au prisme des réseaux ferroviaires », Géocarrefour, n87/2, p.209-224

Desjardins X., Seguret S., et Beaucire F., 2011, « Urbanisation et corridors ferroviaires : quel degré de relation ». In PUMAIN Denise et MATTEI Marie-Flore, Données urbaines $n^{\circ}$ 6, Economica, p.75-80

Dion R.-M., 1974, « Effets des processus volontaristes dans la formation d'une région urbaine : Nancy et les plans d'aménagement et d'extension dans la première moitié du XXe siècle ", Revue Géographique de l'Est, Vol. 14, nº 3-4/ 1974, p.245-311

Dion R-M., 1983, « Le Nouveau Nancy 1870 - 1917 : étude des processus d'urbanisation de deux noyaux primitifs, les quartiers Saint-Léon et Saint-Joseph », in Études d'histoire municipale à la mémoire de Claude Collot, L'urbanisation de Nancy entre 1871 et 1914, Nancy, Publications de l'Université Nancy II, pp. 129 - 246

Franchomme M., Dubois J.-J., 2010, « Documenter les zones humides : vers une meilleure compréhension des paysages d'eau du XIXe au XXe s. », Géocarrefour, Vol. 85/1, p. 7-16.

Flonneau M., Guigueno V., 2009, « De l'Histoire des transports à l'histoire de la mobilité ? Mise en perspective d'un champ ", in De l'histoire des transports à l'histoire de la mobilité ? Etat des lieux, enjeux et perspectives de recherche, Rennes, PU Rennes, pp.11-21

Fritsch B., 2007, « Tramway et prix des logements à Nantes », L'Espace géographique, Tome 36 /2, p. 97-113.

Gallez C., Chardonnel S., 2018, « Comment appréhender les transformations des territoires mobiles ? Des approches circulatoires aux usages de l'accessibilité » In: Talandier M. et Pecqueur B. (dir) Renouveler la géographie économique, Paris, Economica, p. 221-235

Guth S., 2008, Métro et tramway bâtisseurs ? Pas si simple, Predit - Recherches et synthèses, $n^{\circ} 41$, p.2-3

Gregory I., Knowles A., 2011, « Using Historical GIS to Understand Space and Time in the Social Behavioural and Economic Sciences: A White Paper for the NSF, Future Research in the Social, Behavioral \& Economic Sciences, http://www.nsf.gov/sbe/sbe_2020/submission_detail.cfm? upld_id=78

Hamman P ., 2018, « Le tramway en France, des déplacements au projet de ville : dynamiques transactionnelles ", in Gardon S. (dir.), 40 ans de tramways en France, Paris, Editions Libel et Rails \& histoire, p. $213-226$ 
Hecker A., 2018, « Les tramways historiques ont-ils « fait la ville »? Le cas de Nancy », Géotransports, n9, pp. 5-22

IPRAUS-ENSAPB, 2005, Architectures du transport : Rapport final, Vol. 3, Tramway, espaces publics et mobilités, Paris, Predit, 323 p.

Keeney R., 2017, Denver's Historic Streetcar Legacy, DenverUrbanism Blog, https:// denverurbanism.com/denvers-historic-streetcar-legacy (Accessed June 2, 2018)

Laisney F., 2002, «Les Plans d'alignement des villes (1807 - 1819) : une loi mal appliquée », in Lambert-Bresson M. et Térade A. (dir), Villes françaises au XIXème siècle, aménagement, extension et embellissement, Paris, Cahiers de l'IPRAUS, Editions Recherches / IPRAUS, p. 15-29

Laisney F., 2011, Atlas du Tramway dans les villes françaises, Paris, Éditions Recherches, 424 p.

Lambert-Bresson M., 2016, « Implantation des gares, lotissements et émergence des nouvelles centralités entre Asnières et Colombes au XIXè siècle ", in Lambert-Bresson, M., Térade A., 2016, Paysages du mouvement, Paris, Editions Recherches / IPRAUS, 352 p.

Marseille G., 2016, « Une loi pour réformer la planification urbaine Nancy et la loi Cornudet (1919-1949) », Annales de l'Est, Numéro spécial 2016, p. 159-173.

Meunier-Chabert M. et Trêve-Thomas I., 2018, « l'Expérience française du tramway des années 2000 à nos jours : un outil au service d'une meilleure articulation urbanisme - transport », in Gardon S. (dir.), 40 ans de tramways en France, Paris, Editions Libel et Rails \& histoire, p. 119 -130

Merlin P., 1982, Les Transports à Paris et en Ile-de-France, Paris, La Documentation Française, Notes et Études documentaires, $111 \mathrm{p}$.

Maulat J., 2015, « Contractualiser pour coordonner urbanisme et transport ? Regards croisés sur quatre expériences de contrats d'axes ferroviaires ». Flux, $\mathrm{n}^{\circ}$ 101-102, p.82-98

Offner J.-M., 1993, « Les « effets structurants » du transport : mythe politique, mystification scientifique », Espace géographique Vol.22, n 3, p.233-242

Pons F., 2008, Utilisation de données anciennes pour la connaissance des risques de submersions marines, in Colloque, SHF : Nouvelles approches sur les risques côtiers, Paris, 30-31 janvier 2008. En ligne http://cete-aix.fr/dwld/NUNIEAU/2007-11-16-SHF-PONS.pdf

Pumain D., 1982, “Chemin de fer et croissance urbaine en France au XIXe siècle”, Annales de Géographie, Vol. 91, no. 507, p. 529-550

Redondo B., 2012, «Tramway et territoire : quel urbain en perspective ? », Revue Géographique de l'Est, Vol. 52, nº 1-2 2012, 14 p.

Robert J., 1974, Histoire des transports dans les villes de France, Neuilly, compte d'auteur, 529 p.

Schaeffer K. H., Sclar E., 1980, Access for All: Transportation and Urban Growth, Columbia University Press, $182 \mathrm{p}$.

Sicard - Lenattier H., 2000, Les Alsaciens-Lorrains à Nancy entre 1871 et 1914 : Une intégration réussie, Thèse sous la direction de François ROTH, soutenue à l'Université Nancy II, U.F.R. Sciences humaines, 2 tomes, $493 \mathrm{p}$.

Tricoire, J., 2007, Le tramway en France, Paris, La Vie du Rail, 159 p.

Valette P., Carozza J.-M., 2010, « Mise en œuvre d'une démarche géohistorique pour la connaissance de l'évolution des paysages fluviaux : l'exemple de la moyenne vallée de la Garonne ", Géocarrefour, Vol. 85/1, p. 17-27 
Vigarié A., 1983, «Le tramway de Nantes : un exemple d'utilisation des transports en commun pour contrôler et orienter l'urbanisation ", Géocarrefour, n58-1, p.41-50

Warner S.B.J., 1978, Streetcar suburbs, The Process of Growth in Boston, 1870 - 1900, Cambridge et Londres, Harvard University Press, 236 p.

Woessner R., Lanneaux M-A., 2012, « Territoires et Transports en Commun en Site Propre : réalisations, limites et perspectives ", Revue Géographique de l'Est, Vol.52, n¹/2, nn

Zembri P., 2012, « La conception des transports collectifs en site propre (TCSP) en France : des tracés problématiques?", Revue Géographique de l'Est, vol. 52 / 1-2, nn

\section{NOTES}

1. Banque de France, 1872, étude citée par Sicard - Lenattier 2000.

2. Loi de 1919, plan de Nancy approuvé en 1932

3. Loi du 15 février 1902, relative à la protection de la santé publique, qui impose notamment le dépôt en mairie d'un descriptif et de plans des immeubles à construire.

4. La plus grande extension du réseau est visible sur la Figure 4, cf supra

5. La numérotation des lignes de tramway a été très fluctuante, découpant et recomposant régulièrement les sections de part et d'autre du point central). Les lignes "origine/destination » évoluent donc fréquemment, de même que leur identification (numéro, lettre ...). De fait, la numérotation adoptée dans cette recherche s'appuie sur l'ordre de concession de sections entières, indépendamment de tout système officiel, nécessairement circonscrit à une époque précise.

6. Aujourd'hui, l'estimation du périmètre d'attraction du tramway est souvent plus vaste. Il varie selon les auteurs, et peut atteindre $500 \mathrm{~m}$. autour des arrêts. Pour cette étude, le périmètre de $200 \mathrm{~m}$. a été retenu d'une part en raison de la multiplication des lignes, menant à la coalescence de périmètres d'attraction trop élargis, qui brouille l'information. D'autre part, nous souhaitons mettre en évidence l'attractivité particulière du réseau sur les espaces à proximité immédiate, celle-ci déclinant à mesure que l'on s'éloigne de l'infrastructure.

7. «Plan d'extension de la Ville de Nancy dressé par la Société des Architectes de l'Est de la France pour l'exposition de la Cité moderne à Nancy (mai 1913) », reproduit par Dion 1974.

\section{RÉSUMÉS}

Au moment où les principes d'aménagement placent au cœur de la croissance des agglomérations le principe d'une adhérence prioritaire des extensions nouvelles aux axes de transport collectif, un retour sur l'avancée de la tache urbaine au XIXème siècle semble pertinent. Ce moment de forte croissance urbaine est également celui où se constitue le tramway, favorisant, dit-on, une première extension des faubourgs desservis par ces infrastructures nouvelles. La mise en place d'un SIG historique, centré sur la ville de Nancy, apporte des éléments d'éclaircissement sur le rôle joué par ces tramways historiques sur l'organisation de la croissance de la ville, principalement dans espaces péricentraux, de faibles densités. Spontanément ou sous l'action des politiques municipales ou des investisseurs privés, la population s'est-elle concentrée autour de 
ces infrastructures, devenant ainsi des axes amenés à structurer l'agglomération, y compris sur le long terme?

Urban development places the principle of a priority adhesion of the new extensions to the axes of public transport. A return on the progress of the urban fabric in the 19th century seems relevant. At that time, urban growth was particularly strong. It is also the time when tramways were built, promoting a first extension of the suburbs served by this new infrastructure. A historical GIS, focused on the city of Nancy, gives information on the role played by these historical tramways on the growth of the city, mainly in pericentral areas with low densities. Spontaneously or under the action of municipal policies or private investors, has the population really concentrated along these infrastructures, which thereby became structural axes for the city, including in the long term?

In einer Zeit, in der Planungsgrundsätze das Prinzip der vorrangigen Einhaltung neuer Erweiterungen der Achsen des öffentlichen Verkehrs in den Mittelpunkt des Wachstums von Ballungsräumen stellen, scheint eine Rückkehr zum Fortschritt der städtischen Aufgabe im 19. Jahrhundert relevant zu sein. In diesem Moment des starken städtischen Wachstums wird auch die Straßenbahn gebaut, die, wie es heißt, eine erste Erweiterung der von dieser neuen Infrastruktur bedienten Vorstädte begünstigt. Die Einrichtung eines historischen GIS, das sich auf die Stadt Nancy konzentriert, bringt Elemente zur Klärung der Rolle dieser historischen Straßenbahnen bei der Organisation des Wachstums der Stadt, hauptsächlich in perikentralen Gebieten mit geringer Dichte, mit sich . Hat sich die Bevölkerung spontan oder kommunalpolitisch oder von Privatinvestoren auf diese Infrastrukturen konzentriert und wird so zu einer Achse, um die Agglomeration auch langfristig zu strukturieren?

\section{INDEX}

Mots-clés : tramway, forme urbaine, adhérence, effets structurants, SIG historique

Keywords : tramway, urban form, adhesion, structuring effects, historical GIS

Schlüsselwörter : Straßenbahn, städtische Form, Haftung, Strukturierungseffekte, Historisches GIS

\section{AUTEUR}

\section{ANNE HECKER}

Université de Lorraine, LOTERR (EA 7304)

Université de Lorraine - Site de Nancy

CLSH - 23, Boulevard Albert 1er - BP 60446 - 54001 NANCY CEDEX

Anne.hecker@univ-lorraine.fr 